\title{
Travels In Hyper-Diversity: Multiculturalism And The Contextual Assessment Of Acculturation
}

\author{
Marina Doucerain $^{1 *}$, Jessica Dere ${ }^{2,3}$, Andrew G. Ryder ${ }^{1,4}$ \\ ${ }^{1}$ Concordia University (Montreal, Quebec, Canada) \\ ${ }^{2}$ Centre for Addiction and Mental Health (Toronto, Ontario, Canada) \\ ${ }^{3}$ University of Toronto (Toronto, Ontario, Canada) \\ ${ }^{4}$ Jewish General Hospital (Montreal, Quebec, Canada)
}

This is a pre-copyedited, author-produced version of an article accepted for publication following peer review. The final published version is available online with the following doi: 10.1016/j.ijintrel.2013.09.007

\begin{abstract}
ABSTRAC
We argue that current acculturation research offers an incomplete picture of the psychological changes taking place in contemporary multicultural societies. Several characteristics of the Canadian multicultural context highlight the limitations in current acculturation research: namely, themes of hyper-diversity, hybridity, dimensionality and the importance of local context. Canada is a case in point, but these themes are generalizable to other contemporary multicultural contexts. To address the limitations of the traditional psychological acculturation paradigm, we propose an innovative research approach to study acculturation: the Cultural Day Reconstruction Method (C-DRM). We report on two studies that implemented this diary method, to demonstrate that this research tool (1) addresses theoretical critiques of current acculturation research and (2) captures some of the complexity of acculturation in contemporary multicultural contexts. The C-DRM was constructed in response to the local research environment but we hope it will become part of a new generation of tools for the contextual assessment of acculturation.
\end{abstract}

Keywords: multiculturalism, acculturation, methods, Day Reconstruction Method, diary

\section{Introduction}

When our research group started conducting acculturation research in Montreal, we began with the now-standard bidimensional model of orthogonal heritage and mainstream dimensions (e.g., Berry, 2005; Ryder, Alden, \& Paulhus, 2000). Clearly defining the mainstream cultural group turned out to be a thorny issue: both French- and English-Canadian identities could qualify. We temporarily resolved this problem by including two mainstream dimensions, thus creating three-dimensional versions of acculturation instruments (see also Downie, Koestner,

* Correspondence concerning this article should be addressed to Marina Doucerain or Andrew Ryder, Department of Psychology, Concordia University, 7141 Sherbrooke St. W., Montreal, QC, H4B 1R6. email: marinadoucerain@gmail.com or andrew.ryder@concordia.ca. 
ElGaledi, \& Cree, 2004). This seemingly clever solution was short-lived, however, as identifying a single coherent heritage group proved to be just as difficult. "Which one should I use?" was a question we often heard from participants. Picture a migrant born to a Chinese mother and a Spanish father but raised in the Philippines or a French-speaking, Australian-educated, multilingual Tunisian Jewish migrant. At a certain point, one cannot keep adding more subscales to the standard instruments.

Moreover, interpreting the lived experiences of such people through a bidimensional acculturation lens threatens a considerable loss of important information - and we were living our own lives in a multicultural context that reminded us daily that our research methods were insufficient. Montreal's particular complexity urged us to this conclusion, but we came to appreciate that a bidimensional approach to acculturation could only be a beginning for us to properly engage with the complexity of the contemporary multicultural experience. In this paper, we first consider characteristics of the local multicultural context that highlight limitations in current acculturation research. We then propose an innovative method of studying acculturation that was explicitly designed to help address these limitations. Our objective is not to reinvent theories of acculturation; indeed, other acculturation researchers acknowledge many of these same issues in their theoretical work. We hope rather to promote an empirical approach that could help acculturation researchers get closer both to the theoretical possibilities inherent in this theoretical work and the actual lived experience of acculturation.

\section{Multiculturalism In Societies And Minds}

Multiculturalism generally reflects a political ideology supportive of cultural minorities, whereby these groups and their members are not only recognized but also positively accommodated. Multiculturalism is at the heart of a body of political and philosophical work (see e.g., Kymlicka's, 1995, concept of 'group-differentiated rights'), but in practice countries adapt it loosely to fit their own needs. Multiculturalism became official Canadian federal policy in 1971 and part of the Canadian constitution in 1982 (section 27 of the Canadian Charter of Rights and Freedoms). It is based on four core ideas: (1) recognition and maintenance of minorities' cultural heritage; (2) promotion of intergroup communication; and (3) fostering of full participation in Canadian society; with all three points resting to a large extent on (4) the acquisition of at least one of Canada's official languages. Points (2), (3), and (4) reflect Canada's pluralist ideology (Bourhis, 2001) and promote a synthetic, unified society, formed from equally valued and differentiated pieces.

The extent to which these ideals 'work' in Canadian society is beyond the scope of this article. We are instead describing a set of ideals. These ideals nonetheless set Canada's multicultural policy apart from many countries' versions - especially that of most European nations - by rejecting the view that, "society should be divided into separate and disconnected ethnic groups, each with its own territorial spaces, political values and cultural traditions." (Banting \& Kymlicka, 2010, p. 45) Critics such as Banting and Kymlicka (2010) have linked this latter approach to ghettoization of immigrants, increased discrimination, and political radicalism in many European countries.

This account of multiculturalism at the societal, macro-level is interesting to us inasmuch as it permeates and shapes 'micro-level multiculturalism' - the multicultural mind. Indeed, state integration policies provide a social climate that influences individual attitudes and behaviours (Bourhis, Moïse, Perreault, \& Senécal, 1997). More broadly, our theoretical stance toward interactions between macro- and micro-levels is one of mutual constitution between culture, mind, and brain (Ryder, Ban, \& Chentsova-Dutton, 2011; see also Shweder, 1990). We are interested here in the psychological consequences of living in a sociopolitical context such as Canada, especially in the local context of Montreal. Specifically, we focus on the development of the multicultural mind: what are the changes in cognitions, behaviours, motivations, emotions, and identities that take place when a person lives in a complex multicultural environment?

These questions are almost isomorphic with the definition of psychological acculturation (hereafter simply 'acculturation') when applied to a multicultural context. Indeed, acculturation has been defined as: 
.... a process that is executed by an agentic individual ... after meeting and entering a cultural community that is different from the cultural community where he or she was initially socialized. Acculturation involves a deliberate, reflective, and, for the most part, comparative cognitive activity of understanding the frame of references and meanings with regard to the world, others, and self that exist in one's 'home' cultural community and which one has discovered in a new cultural community. This process emerges within the context of interactions, both physical and symbolic, with the members of the 'home' and new cultural communities. Acculturation is an open-ended, continuous process that includes progresses, relapses, and turns (Chirkov, 2009a, p. 94).

Consequently, we would expect the acculturation literature to be the ideal source of answers to questions about the multicultural mind. We will argue, however, that research that fully engages with the ideological and practical consequences of multiculturalism must proceed differently than much of the research found in the existing acculturation literature. To that end, we will first briefly review the dominant paradigm in contemporary acculturation research, and then discuss critiques of this paradigm that are informed by multiculturalism in Canada.

\section{Acculturation And The Contemporary Multicultural Critique}

For the last few decades, acculturation research has been dominated by the framework developed by Berry and colleagues (Ward \& Kus, 2012) and the concept of 'acculturation strategies' (e.g., Berry, 2005). Berry posits two dimensions that define how people go about negotiating the acculturation process: (1) people's attitudes toward cultural heritage maintenance; and (2) people's attitudes toward contact with and participation in the mainstream cultural group. Crossing these orthogonal dimensions yields four acculturation strategies: integration (a relative preference for both heritage maintenance and mainstream contact); separation (a preference for heritage maintenance and no involvement with the mainstream group); assimilation (positive attitudes toward contact with and participation in the mainstream group and a lack of interest in heritage maintenance); and marginalization (a disengagement from both heritage and mainstream cultural concerns). In an expansion of this model, developed in the Quebec context, Bourhis and colleagues identify a fifth possible strategy: individualism, or a rejection of group categories and a preference for treating people as individuals (Bourhis et al., 1997).

An important contribution of this framework is the decisive move away from a unidimensional approach in the international acculturation literature. Indeed, many specific bidimensional approaches to acculturation have been developed and used, including within our research group (e.g., Dere, Ryder, \& Kirmayer, 2010; Ryder et al., 2000; Ryder, Alden, Paulhus, \& Dere, 2013). Even though these approaches do not necessarily use Berry's specific labels and measurement tools, they posit two key dimensions, measure them in a trait-like way using self-report measures, and use them to predict variables of interest-particularly psychosocial adjustment (Arends-Tóth \& van de Vijver, 2006; Chirkov, 2009b). This research tends to emphasize 'acculturative stress' within a general stress-coping perspective (Cabassa, 2003). At least when Berry's four strategies are used, a large number of studies find integration to be the most adaptive strategy (Berry \& Sam, 1997).

\section{Multiculturalism: Challenges To Existing Acculturation Research}

In recent years, this dominant paradigm has faced an increasing number of critiques (e.g., the introduction to a special issue on the topic in this journal; Chirkov, 2009b). A comprehensive review of these critiques is beyond the scope of this paper. Instead, we will focus first on three challenges to acculturation research that stem from characteristics of multiculturalism in Canada. We will then present an innovative approach to the study of acculturation - the Cultural Day Reconstruction Method - that was inspired by our multicultural context and that seeks to address some of the difficulties of acculturation research in complex multicultural contexts. 


\section{Dimensionality: Beyond the Traditional Heritage-Mainstream Dichotomy}

'Hyper-diversity' (Kirmayer, 2013) or 'super-diversity' (Vertovec, 2007) are apt descriptors of the cultural make up of Montreal and Toronto in Canada, and certain large cities in other multicultural countries. Distinct cultural groups are numerous, and diversity arises not only in terms of countries of origin, but also according to language, religion, migration channel, immigration status, gender, age, and level of transnationalism. As a result, the traditional heritage-mainstream dichotomy characteristic of acculturation research is rendered obsolete.

Our own local context in Montreal serves as one striking example. In addition to the diversity typical of larger Canadian cities, language considerations mean that both French- and English-speaking Canadians are effectively mainstream groups. Whereas the former group is a minority in Canada, the latter group is a minority in the province of Quebec. The focus of Canada's multiculturalism policy on intergroup sharing and communication compounds challenges to heritage/mainstream dichotomies by cultivating hybridization between cultural groups. Hybridity includes within-generation instances of cultural syncretism, such as Latino rock or Mandarin pop music, as well as intergenerational mixing of cultural heritages through intermarriages, leading to the emergence of a large number of people for whom hybridity is an experiential, embodied reality.

Scholars have started to contest acculturation bidimensionality by positing what could be called fusion models (Arends-Tóth \& Van de Vijver, 2004). From this perspective, people need not be confined to neat heritage versus mainstream distinctions and are likely to idiosyncratically create altogether new cultural affiliations that mix and combine aspects of relevant cultural groups. Unfortunately, this process of cultural recombination, which Hermans and Kempen (1998) call 'hybridization', is largely absent from empirical examinations of acculturation (Arends-Tóth \& Van de Vijver, 2004). The method proposed here takes a step in correcting this situation by allowing various forms of hybridity.

\section{Situatedness: Domain Specificity and the Importance of Local Context}

Several scholars have underscored the important role that contextual factors such as the socio-political orientation of the mainstream ('host') community (Bourhis et al., 1997), or colonial histories and diasporic communities (Bhatia \& Ram, 2009), play in shaping the multicultural mind. These authors are mostly concerned with the 'macro-context' of acculturating individuals, but we argue that acculturation research should also consider the 'micro-context': namely, the immediate, concrete, local conditions of daily life. People experience macro-influences such as language ideologies and political orientations toward immigrants through daily social interactions. In other words, macro-contextual influences permeate, are enacted in, and are experienced in the micro-context. This theoretical emphasis on 'dailiness' has been emphasized by scholars in cultural studies, such as Certeau (1988) and Lefebvre (2002; see also Highmore, 2002; Moran, 2005). Vertovec (2007) echoes the importance of this perspective by arguing that understanding what he calls 'super-diversity' requires the study of highly local contexts.

In addition, the four requirements of Canadian multiculturalism - heritage maintenance, intergroup communication, full participation in society, and knowledge of at least one official language - form an ambitious cultural program for any person. It is unlikely that all four are salient in all contexts and at all times. Certain cultural components may be salient in specific contexts according to systematic patterns. This aspect of multiculturalism highlights the issue of domain specificity (Dere et al., 2010). In recent years, an increasing number of scholars have demonstrated that acculturation is domain-specific and that a person's preferences and attitudes with respect to cultural groups can vary across life domains. For example, Arends-Tóth and van de Vijver (2004) found that Turkish immigrants in the Netherlands favoured different acculturation strategies across public and private life domains. Similarly, Lechuga (2008) showed that acculturation scores were susceptible to cultural priming effects. This superordinate level of specificity can be extended to variation across specific situations. For instance, Clément and Noels' (1992) work on situated identity revealed that the expression of ethnolinguistic identity displayed important inter-situational variation. In a related vein, Hong and colleagues 
(Hong, Morris, Chiu, \& Benet-Martínez, 2000) showed that biculturals switched between different cultural frames in response to cultural icons. More work on domain- and context-specific aspects of acculturation is needed to understand how multicultural people navigate their complex cultural environments.

Both considerations of context and of domain-specificity underscore the situated nature of acculturation and emphasize the important role that the environment plays in shaping and modulating the acculturation process. The method proposed here allows researchers to take into account the environment that acculturating individuals navigate and thus to focus on the concrete nature of their lived experience.

\section{Behaviour: Attitudes are not Actions}

The core ideas of multiculturalism in Canada described earlier are prescriptive: they define a set of ideals thought to foster a just, peaceful, and tolerant society. This prescriptive side of multiculturalism, however, begs examination of its descriptive facet. How do these principles trickle down to the level of daily behaviours and interactions? Do multicultural people actually use an official language in their daily lives? Do they make choices that maintain their cultural heritage? What do their social networks look like? In other words, how do multicultural principles translate into multicultural realities?

These fundamental questions are not fully addressed by existing acculturation research, and we question whether current methods could ever provide satisfactory answers. The emphasis on acculturation strategies and related self-report questionnaires represents an emphasis on ideals at the personal level, but not on actual behaviours. In their Relative Acculturation Extended Model, which is an expansion of the dominant bidimensional model, Navas and colleagues (Navas et al., 2005) stress the need to distinguish between ideal or preferred acculturation strategies and those adopted in reality. Similarly, a number of critics have called for measures that characterize multiculturals' behaviours, arguing that, "the uniformly high scores of integration ... are research artifacts, based on a high appreciation of biculturalism and bilingualism, which correspond neither with measures of bicultural/bilingual knowledge nor with practices" (Boski, 2008, p. 145). We could not agree more with this position. Indeed, Ward and Kus (2012) recently showed that an integration strategy is more likely to be endorsed when research instruments are based on attitudes as compared to self-reported behaviours. It is time acculturation research embraced the study of actual behaviours. The method proposed here, which includes a diary instrument, represents a step in this direction by examining participants' lived experiences. It is to this method that we now turn.

\section{The Cultural Day Reconstruction Method (C-DRM)}

We have argued that our Canadian multicultural context foregrounds three areas of relative neglect in traditional acculturation research - dimensionality/hybridity, situatedness, and concrete behaviours. Our goal is now to present a cultural adaptation of the Day Reconstruction Method (C-DRM; Kahneman, Krueger, Schkade, Schwarz, \& Stone, 2004), a method designed to address these weaknesses. We will then present data from two studies that have used this method. Initial work on the C-DRM (Watanabe \& Ryder, 2007) was driven by a desire to address the marked disconnect between the complexity of the acculturation process and the simplicity of most measurement tools in this field. The goal was to develop an instrument that would be more clearly grounded in the lived reality of respondents, that would be easily adaptable for multiple cultural reference groups and a range of acculturation experiences, and that could yield both quantitative and qualitative data.

The C-DRM was inspired by the work of Kahneman and colleagues (Kahneman et al., 2004), who developed the original DRM, a well-validated assessment of daily activities in which the preceding day is divided into episodes that are each rated in terms of activity, social interaction, and emotional valence. This method was designed to provide data that are similar to those collected using experience sampling methodologies, but in a more efficient and less costly manner (Kahneman et al., 2004). To date, the DRM has primarily been used in well-being research (e.g., Knabe, Rätzel, Schöb, \& Weimann, 2010; Kopperud \& Vittersø, 
2008), with no particular attention paid to cultural identity or cultural change. In its assessment of everyday experiences in context, this method provided an intriguing possibility for acculturation research.

The C-DRM involves modifications to the original DRM that allow for the assessment of a number of factors relevant to cultural identification and acculturation. To begin, participants list the cultural groups with which they identify. This first page in the C-DRM is known as the 'culture sheet', and is not part of the original DRM. In addition to 'mainstream' (e.g., English-Canadian, French-Canadian) and 'heritage' (e.g., Chinese, Haitian) groups - with room for more than one group per category - participants are also encouraged to include 'hybrid' cultural groups (e.g., Chinese-Canadian), religious or spiritual affiliations, and any other salient cultural identities. Working definitions are provided to assist participants. For example, for hybrid cultural groups: "People may endorse attitudes, values, and practices that incorporate elements of two or more cultures and which result in a new and unique hybrid culture, reflective of the prolonged contact between the groups in question". This initial step already places the C-DRM apart from the majority of current acculturation measures, by allowing for the inclusion of numerous and varied cultural reference groups.

Participants next divide up their previous day into a series of episodes, similar to a sequence of scenes in a film, using the 'episode sheets'. Once the episodes have been listed, participants respond to a series of questions for each one. The key modification to the original DRM is the inclusion of several questions regarding cultural identification. For each episode, participants are asked to indicate their cultural affiliation(s), by drawing on their completed culture sheet, along with language(s) used and the cultural backgrounds of people with whom they were interacting, if applicable. See Figure 1 for an example of a completed episode sheet. Finally, participants report their feelings during the episode, by indicating the extent to which they experienced a series of affect descriptors (e.g., happy, competent/capable, worried/anxious).

\section{Study 1}

The goal of this preliminary study was to determine whether or not the Culture-DRM yields data that cannot be captured by traditional acculturation measures. Because of its exploratory nature, we did not formulate specific research questions or hypotheses. We simply examined the diversity of cultural groups with which participants affiliated and how these affiliations were used throughout the day.

\section{Methods}

One hundred and nineteen undergraduate students at Concordia University, an English-language university in Montreal, were recruited through flyers posted in culturally-defined or international student associations (e.g., Chinese Students' Association, International Students' Association, etc.). In this study, only migrants were used: participants were included in the final sample if they had arrived in Canada less than ten years prior to the study date. The final sample included 29 women and 27 men with an average age around 24 years $(\mathrm{M}$ age $=24.12$, SD $=5.63)$, who had lived in Canada for an average of around three years $(\mathrm{M}=3.13, \mathrm{SD}=2.12)$. The sample represented a variety of cultural origins, especially Arabic-heritage (40\%) and Chinese-heritage (27\%). After providing written informed consent, participants filled out a paper-and-pencil version of the C-DRM.

\section{Results \& Discussion}

As this study marked the initial examination of the C-DRM, the statistical analyses were largely descriptive. The first set of results involved the number of cultural identities listed by participants. They reported an average of nearly five identities in total $(\mathrm{M}=4.50, \mathrm{SD}=1.32)$. On average, participants reported more than one heritage identity $(\mathrm{M}=1.21, \mathrm{SD}=.41)$ and more than one hybrid identity $(\mathrm{M}=1.39, \mathrm{SD}=.91)$. In addition, $25 \%$ of the hybrid identities listed by participants did not involve the combination of a heritage and a mainstream identity, but rather various other two-identity combinations. Furthermore, $69 \%$ of the sample reported at least one 
religious/spiritual identity.

\section{First Morning Episode}

Please look at you Diary and select the earliest episode you noted in the Morning.

This is episode number $1 \mathcal{M}$, which began at $7: 30$ and ended at 8:15 .

1. What were you doing? (check all that apply)

commuting

studying

doing housework

$\checkmark$ eating

$\underline{\checkmark}$ socializing

_ nap / resting / relaxing

computer / internet / e-mail

intimate relations

shopping

working

$\checkmark$ preparing food: what kind? Canadian

taking care of your children

praying / worshipping / meditating

watching television (TV)

reading newspaper / book / magazine

on the phone

exercising

other; please specify:

1a. If applicable, what was the main language used during the activity? (e.g. watching TV in Spanish, online chat in Chinese) English
2. Where were you?

at school $\quad \checkmark$ at home__ at work_

3. Were you interacting with anyone?

(e.g. in person, on the phone, internet chat - text and/or video, etc.)

$\checkmark$ Yes__ No if no one, skip to Question 4.

3a. If you were interacting with someone (please check all that apply)

__ spouse / significant other __ my child / children

parent(s)/relative (s)_ friend(s)

_ classmate(s) _ co-worker(s)

_other: roommates

Their cultural background

$\checkmark$ English-Canadian _ French-Canadian

Someone / people from my heritage culture

Other:

3b. In what language were you primarily interacting?

My heritage language $\quad \checkmark$ English _ French

_ Other (please specify):

4. During this episode, what was your cultural affiliation / orientation?

Please refer to Packet 2 (your Culture Sheet) and enter a cultural affiliation/orientation (and corresponding number) for this episode. (e.g., Number: 4 Affiliation/orientation: Chinese-Canadian)

Number: 1 Affiliation/orientation: English-Canadian

\section{Figure 1 | A sample episode of the DRM, with fictional answers}

We then examined how participants described their day in order to see whether the identities listed on the culture sheet were actually used on the episode sheets. On average, participants reported ten episodes $(\mathrm{M}=10.15, \mathrm{SD}=$ $3.19)$, and they used nearly three distinct identities in describing the course of their day $(\mathrm{M}=2.76, \mathrm{SD}=1.08)$. Moreover, we found that on average participants switched between different cultural identities four times during the day $(\mathrm{M}=4.00, \mathrm{SD}=2.87)$.

The results presented here should be seen as largely preliminary, illustrating the potential utility of the CDRM for acculturation research. They suggest that the C-DRM can account for cultural identities that traditional instruments generally cannot, highlighting the limitations of bidimensional measures that pose questions about only two (or perhaps three) pre-specified cultural groups. Our results also revealed that the C-DRM taps into the notion of hybrid identities in ways that are impossible for most acculturation measures. The finding that a fair proportion of hybrid identities did not involve a mainstream-heritage combination stands in contrast to the often implicit assumption in the acculturation literature that hybrid identities represent the merger of a mainstream identification (e.g., Canadian) and a heritage one (e.g., Lebanese). This finding fits with the earlier discussion regarding hybridity and emphasizes the importance of remaining grounded in participants' lived experiences.

The finding that participants switched cultural affiliations several times during the day is consistent with the work of Hong, Benet-Martinez and others (e.g., Benet-Martínez, Leu, Lee, \& Morris, 2002; Hong et al., 2000), and supports the notion that local contextual factors play a crucial role in scaffolding momentary cultural experiences. Which factors are implicated remains an open question, however. Also unresolved is the relation 
between these fluctuating, momentary, context-bound cultural affiliations and people's more general, stable acculturation preferences as measured by traditional acculturation self-report questionnaires. We believe that the $\mathrm{C}$-DRM is able to provide answers to both of these questions, as we demonstrate in Study 2.

\section{Study 2}

This study built on the results found in Study 1 by focusing on the relations between local context and cultural affiliation; by examining how general acculturation preferences (as measured by traditional acculturation tools) influence these relations; and by taking into account hybrid affiliations. Specifically, we sought to answer the following questions:

(1) What contextual elements in the daily life of multicultural individuals predict momentarycultural affiliation?

(2) What is the influence of general acculturation attitudes (toward heritage and mainstream cultural groups) on momentary cultural affiliation?

These questions were informed by our stance toward mainstream acculturation research. Note that while our work attempts to distance itself from the dominant acculturation paradigm, it aims to complement rather than reject it. We believe that general attitudes toward one's cultural groups do play a role and influence the acculturation process as it is enacted in daily life. In other words, macro-preferences are likely to permeate micro-choices. Because of the exploratory nature of the research presented here, we did not formulate specific hypotheses. We simply expected that some contextual aspects of the immediate environment would predict momentary cultural affiliation and that general acculturation-related attitudes would influence momentary cultural affiliation.

\section{Methods}

Participants and procedure. One hundred and eleven multicultural students at Concordia University, an English-speaking university in Montreal, took part in the study. Participants were recruited in classes and through flyers posted in culturally-defined or international student associations (e.g., Chinese Students' Association, International Students' Association, etc.). We screened the participants for inclusion in the final analysis on the basis of their cultural background. Specifically, only participants who reported at least one cultural identity in addition to Canadian, English-Canadian, French-Canadian, and/or Quebecois in their culture sheet were included. This procedure eliminated ten participants. The final sample comprised 69 women and 32 men with an average age around 24 years $(\mathrm{M}=24.09, \mathrm{SD}=6.10)$. The majority of participants were first generation immigrants (73\%) who had lived in Canada for an average of around four-and-a-half years $(\mathrm{M}=4.52$, $\mathrm{SD}=7.23)$. The sample represented a variety of cultural origins, especially Arabic-heritage (13\%) and Chineseheritage (24\%). For example, $24 \%$ of participants were of Chinese descent and 13\% were of Arabic-speaking heritage. After giving written informed consent, participants filled out paper-and-pencil versions of the C-DRM (see earlier section for a description and Figure 1 for a sample episode) and of a Quebec-specific version of the Vancouver Index of Acculturation (VIA; Ryder, Alden, \& Paulhus, 2000).

The Quebec-specific version of the VIA is a 30-item self-report measure with three parallel subscales assessing acculturation towards the heritage group (VIA-H), acculturation towards the French-Canadian mainstream group (VIA-FC), and acculturation towards the English-Canadian mainstream group (VIA-EC). These last two subscales reflect the complex nature of the mainstream cultural context in Montreal. A sample item is, "I would be willing to marry a person from my heritage culture". The cultural referent changes to "an English-Canadian person" and to "a French-Canadian person" for the VIA-EC and VIA-FC subscales, respectively. Participants rate their agreement to items on a 9-point Likert-type scale, with higher scores indicating more agreement. Internal consistencies were adequate to high in our sample (VIA-H: $\alpha=.86$; VIA-EC: 
$\alpha=.77$; VIA-FC: $\alpha=.87$ ). A single 'acculturation toward the mainstream group' subscore (VIA-M) was obtained by computing the higher value between scores on the VIA-EC and the VIA-FC for each participant.

Coding. Several variables characterizing the episodes had to be recoded to be amenable to analysis. "Language used" was recoded into four categories: Mainstream (i.e., English or French), heritage (e.g., Spanish or Chinese), bilingual (use of more than one language during the episode), and no interaction. "Cultural background of interlocutor" was also recoded into four categories: mainstream (e.g., Canadian or Quebecois), heritage (e.g., Egyptian), hybrid (e.g. Italian-Canadian), and no interaction. In terms of activities carried out during the episode, we focused on whether or not that activity was related to food (e.g., eating or cooking). Two considerations motivated this choice: (1) many acculturation scales include food and cuisine as a culturally relevant domain; and (2) food has been identified as a central cultural symbol (Cleveland, Laroche, Pons, \& Kastoun, 2009). "Activity" was therefore recoded into two categories, food-related and not food-related. Note that future researchers could choose other types of activities listed in the episode sheets for similar attention. Cultural affiliation, as our criterion variable, was recoded into only three categories: Mainstream, hybrid, and heritage. Instances that listed religious groups were recoded as heritage affiliations.

Analysis. Because each participant yielded a cluster of data points (episodes nested within individuals), we used multilevel modelling to analyze the data. This procedure accounts for interdependence among observations within each participant and allows us to model between-person variability in the relationships between microvariables and momentary cultural affiliation. In addition, this analysis strategy is in keeping with recent theoretical developments that advocate multilevel analyses in cross-cultural psychology (van de Vijver, van Hemert, \& Poortinga, 2008).

Our analysis predicted momentary cultural affiliation from two levels of predictors. First level predictors, or micro-variables, characterized the local, momentary context. These values changed from episode to episode. Second level predictors, or macro-variables, characterized participants' acculturation attitudes. These values were invariant across episodes for a given participant.

Specifically, given our binary dependent variables (affiliation to one group vs. the other) we fitted two generalized linear mixed models (logistic) models to the data. The first model contrasted mainstream affiliation vs. hybrid and heritage affiliations grouped together by estimating the probability of reporting hybrid/heritage affiliation for each episode. The second model contrasted hybrid vs. heritage affiliations by estimating the probability of reporting heritage affiliation for each episode ${ }^{1}$. For both models, we first tested the null hypothesis that random effects equal zero through 3000 bootstrapped estimations of the null model. Rejecting the null hypothesis confirms that observations within a person are interdependent and justifies using multilevel modeling over a simple logistic regression approach. The null model also allows us to estimate within- and betweenperson variance and it serves as a baseline against which we will compare more complex models.

We entered predictors in three sequential steps: (1) micro-variables characterizing structural aspects of the context (presence of food-related activity and location); (2) micro-variables characterizing social aspects of the context (language used with interlocutor and cultural background of interlocutor); and (3) macro-variables characterizing individuals' acculturation to mainstream and heritage groups (scores on VIA-H and VIA-M). All models included only random intercepts ${ }^{2}$. Fixed effects coefficients will be presented only for the full model. Ninety-five percent confidence intervals were obtained for these coefficients based on 1000 bootstrapped estimates.

1 We used this nested approach rather than a multinomial logit random effects model because these latter models are much more complex to evaluate, as they require evaluation of multidimensional integrals (Malchow-Møller \& Svarer, 2003). For that reason, they are still the source of active research (Hartzel, Agresti, \& Caffo, 2001). The two models were fitted to the data by Laplace approximation, using the lme4 package in R version 2.12.1 (Bates, 2011).

2 At the episode level, the probability $p_{\mathrm{ij}}$ of affiliating to the heritage cultural group for person $j$ during episode $\mathrm{i}$ was modeled according to the following level 1 equation: 
At each step, change in model fit was assessed in several ways. First, we computed the Somer's D statistic (Somers, 1962) for each model as a measure of ordinal association. Second, we computed the reduction in variance of intercept random effect (relevant for macro-variables only) (Singer, 1998, p. 332), which provides an R2-type measure of improvement in model fit and is typically used in multilevel analysis (Singer \& Willett, 2003). This measure indicates the extent to which person-level variables explain random variation around the intercept. Third, we compared changes in information criteria using the Akaike Information Criterion (AIC) and Bayesian Information Criterion (BIC), and computed likelihood ratio tests.

Table 1 | Model Fit in Predicting Mainstream vs. Heritage/Hybrid Affiliation

\begin{tabular}{|c|c|c|c|c|c|c|c|}
\hline Model & $\tau_{00}^{\wedge}$ & Somers' D & AIC & BIC & loglik & $\chi_{\text {diff }}^{2}(d f)$ & $\mathbf{p}$ \\
\hline Null model & & & 658.6 & 667.2 & -327.3 & & \\
\hline 1. Structural context & & .80 & 616.8 & 642.6 & -302.4 & & \\
\hline $\begin{array}{l}\text { Difference between model } 1 \text { and null } \\
\text { model }\end{array}$ & & & & & & $49.84(4)$ & $<.001$ \\
\hline 2. Social context & 3.15 & .88 & 531.0 & 582.7 & -253.5 & & \\
\hline $\begin{array}{l}\text { Difference between model } 2 \text { and } \\
\text { model } 1\end{array}$ & & & & & & $97.79(6)$ & $<.001$ \\
\hline 3. Acculturation scores & 2.78 & .88 & 522.0 & 582.4 & -247.0 & & \\
\hline $\begin{array}{l}\text { Difference between model } 3 \text { and } \\
\text { model } 2\end{array}$ & & & & & & $12.93(2)$ & .002 \\
\hline
\end{tabular}

Note. $\mathrm{AIC}=$ Akaike Information Criterion; BIC = Bayesian Information Criterion; loglik = log likelihood. The introduction of macro-variables reduced intercept variance by $12 \%$.

\section{Results \& Discussion}

Results are presented in three sections: (1) general descriptive results for the DRM; (2) results from the first model, predicting mainstream vs. heritage and hybrid together affiliations; and (3) results from the second model, predicting hybrid vs. heritage affiliations.

Descriptive results. On average, participants listed almost five different cultural identities $(\mathrm{M}=4.93, \mathrm{SD}=$ 1.52) on the culture sheet. They mentioned more than one cultural identity for the mainstream, heritage, and

$$
\begin{gathered}
\text { culture. } \int \dot{c}{ }_{i j}+r_{i j} \text { ewherer }_{i j} N\left(0, \sigma^{2}\right) \\
\ln \left(\frac{p_{i j}}{1-p_{i j}}\right)=\beta_{0 j}+\beta_{1 j} \text { food }_{i j}+\beta_{2 j} \text { location }_{i j}+\beta_{3 j} \text { language }_{i j}+\beta_{3 j} \dot{c}
\end{gathered}
$$

and where $\beta_{0 \mathrm{j}}$ represents the expected log-odds of affiliating to the heritage group when all predictors are set to their reference level, $\beta_{\mathrm{xj}} \mathrm{X}_{\mathrm{ij}}$ represents the change in log-odds of affiliating to the heritage group as a function of scores on micro-predictor $\mathrm{X}$, and $r_{\mathrm{ij}}$ represents the error term associated with episode $\mathrm{i}$ for person $\mathrm{j}$.

At the person level, the episode-level intercept and slopes were modeled according to the following level 2 equations:

$$
\begin{gathered}
\beta_{0 j}=\gamma_{00}+\gamma_{01} \text { VIA. } M_{j}+\gamma_{02} \text { VIA. } H_{j}+u_{0 j} \text { espwhere } u_{0 j} N\left(0, \tau_{00}\right) \\
\beta_{1 j}=\gamma_{10} n n \beta_{2 j}=\gamma_{20} n n \beta_{3 j}=\gamma_{30} n n \beta_{4 j}=\gamma_{40}
\end{gathered}
$$

and where $\gamma_{00}$ is the overall intercept, $\gamma_{0 \mathrm{x}} \mathrm{X}$ is the effect of macro-predictor $\mathrm{X}$, and $u_{\mathrm{oj}}$ represents the unique effect of person $\mathrm{j}$ on the intercept. 
hybrid categories $(\mathrm{M}=1.59, \mathrm{M}=1.43$, and $\mathrm{M}=1.14$, respectively). Of these, more than two $(\mathrm{M}=2.24, \mathrm{SD}=$ 1.07) were actually used during the day, as reported in the episodes that had a particular cultural affiliation. Participants reported an average of more than five-and-a-half episodes $(\mathrm{M}=5.54, \mathrm{SD}=1.52)$ that had a particular cultural affiliation. The average duration of an episode was 78.51 minutes. In total, 551 episodes were analyzed. Among these, 194 referred to a mainstream affiliation, 252 to a heritage affiliation, and 105 to a hybrid affiliation.

Table 2 | Fixed Effects and Random Effects for the Full Model Predicting Mainstream vs. Heritage/Hybrid Affiliation

\begin{tabular}{|c|c|c|c|c|c|c|}
\hline Parameter & Adjusted OR & Estimate & SE & $95 \% \mathrm{Cl}$ & $\mathbf{z}$ & $\mathbf{p}$ \\
\hline \multicolumn{7}{|l|}{ Random effects } \\
\hline Intercept Variance & & 2.77 & & & & \\
\hline \multicolumn{7}{|l|}{ Fixed effects } \\
\hline Intercept & 7.42 & 2.01 & 1.64 & {$[-0.66,5.09]$} & 1.21 & .23 \\
\hline \multicolumn{7}{|l|}{ Level 1 (episodes) } \\
\hline Food-related activity yes & 2.24 & 0.81 & 0.32 & {$[0.23,1.49]$} & 2.53 & .01 \\
\hline Location school & 0.23 & -1.49 & 0.40 & {$[-2.15,-0.89]$} & -3.71 & $<.001$ \\
\hline Location work & 0.30 & -1.21 & 0.78 & {$[-2.72,0.03]$} & -1.56 & .12 \\
\hline Location other & 0.33 & -1.10 & 0.36 & {$[-1.84,-0.41]$} & -3.07 & .002 \\
\hline Language heritage & 27.09 & 3.30 & 0.93 & {$[2.30,17.27]$} & 3.55 & $<.001$ \\
\hline Language bilingual & 2.79 & 1.03 & 0.58 & {$[0.26,2.28]$} & 1.77 & .08 \\
\hline Language no interaction & 0.52 & -0.65 & 0.59 & {$[-1.79,0.35]$} & -1.12 & .26 \\
\hline Interlocutor heritage & 13.74 & 2.62 & 0.50 & {$[1.79,3.65]$} & 5.23 & $<.001$ \\
\hline Interlocutor hybrid & 3.51 & 1.26 & 0.44 & {$[0.64,1.98]$} & 2.80 & .005 \\
\hline Interlocutor none & 4.00 & 1.39 & 0.60 & {$[0.51,2.47]$} & 2.31 & .02 \\
\hline \multicolumn{7}{|l|}{ Level 2 (participants) } \\
\hline VIA-M & 0.44 & -0.81 & 0.24 & {$[-1.28,-0.46]$} & -3.42 & $<.001$ \\
\hline VIA-H & 1.61 & 0.47 & 0.19 & {$[0.16,0.86]$} & 2.48 & .01 \\
\hline
\end{tabular}

Note. $\mathrm{OR}=$ odds ratio; $\mathrm{Cl}$ = confidence interval; interlocutor = identified cultural group of other person(s) in the interaction.

Predicting mainstream vs. heritage/hybrid affiliation. Incremental changes in model fit as a result of hierarchical entry of predictors are presented in Table 1. Each step produced both a decrease in information criteria values and a statistically significant chi-square value for likelihood ratio tests, indicating that the introduction of each block of variables increased model fit. The high Somers' D final value also showed that the model was effective in accurately predicting cultural affiliation. In other words, characterizing the structural context, the social context, and general acculturation attitudes contributed to this prediction. Moreover, the introduction of macro-variables accounted for an appreciable proportion of intercept variance among individual participants. These results converge to show that the selected variables were successful in predicting mainstream versus heritage/hybrid cultural affiliation in this sample, supporting the hypothesis that contextual aspects of the immediate environment predict momentary cultural affiliation and that general acculturation-related attitudes also influence this affiliation. 
Table 2 reports the regression results for the full model. In accordance with measures of model fit, all variables significantly predicted cultural affiliation. For ease of interpretation, the relation between levels of each variable (including all reference levels) and cultural affiliation is shown in Figure 2. In this figure, regression coefficients have been transformed back to probabilities of reporting a mainstream cultural affiliation versus a heritage/hybrid cultural affiliation. In other words, smaller values indicate a greater probability of reporting affiliation to a mainstream cultural group, and a value of .5 indicates equal probability of reporting affiliation to either group. Overall, Figure 2 shows that a higher probability of reporting a heritage/hybrid affiliation during an episode is associated with the presence of a food-related activity, home location, heritage language of interaction, heritage culture of interlocutor, lower VIA mainstream scores, and higher VIA heritage scores. Interestingly, bilingual interaction and interaction with a hybrid-culture interlocutor are associated with probabilities that fall in-between those associated with the mainstream and heritage poles. These results provide initial support for the idea that hybrid cultural elements are distinct from both mainstream and heritage components.
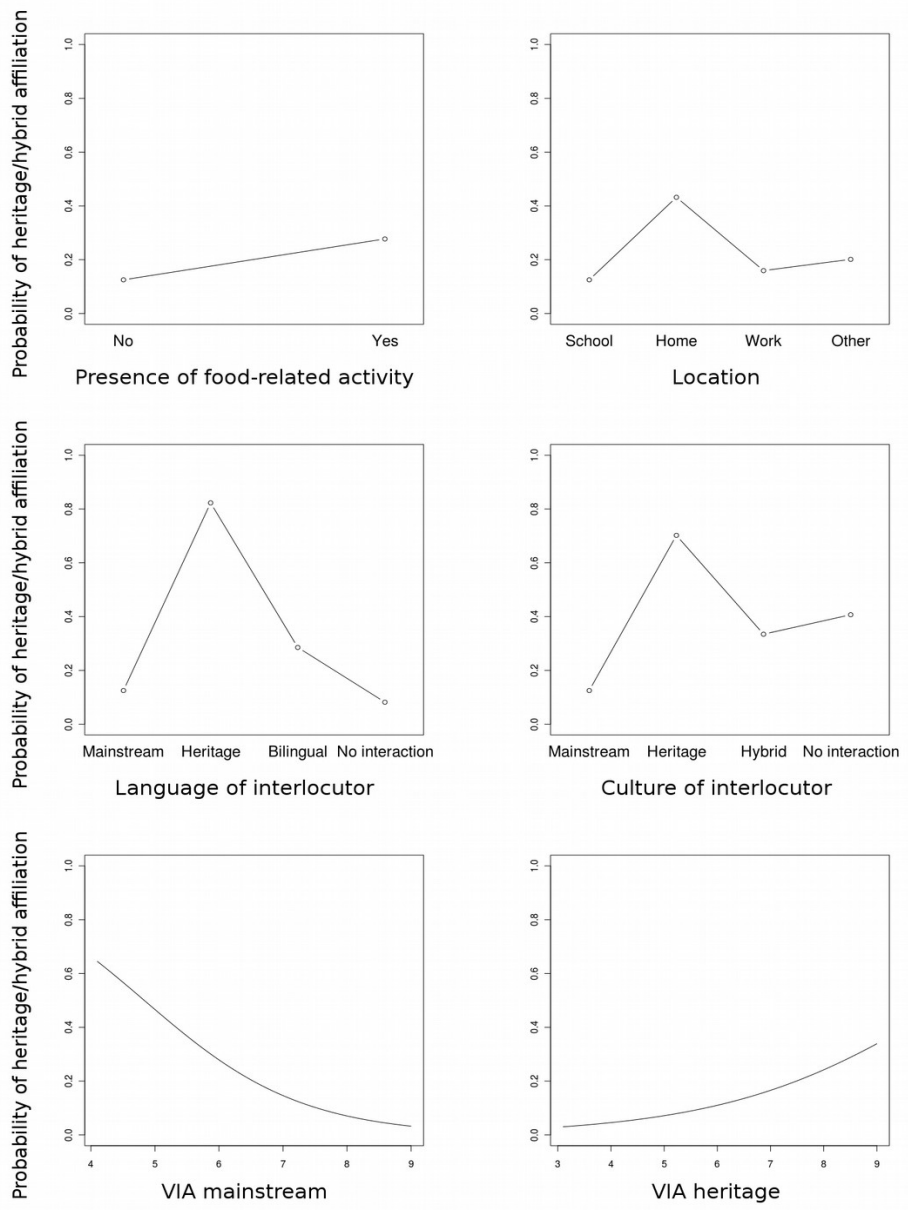

Figure 2 | Probability of affiliating to mainstream vs. heritage/hybrid cultural groups

The reference levels are: No food-related activity, home location, mainstream language of interaction, mainstream culture of interlocutor, mean VIA mainstream score $(\mathrm{M}=6.69)$, and mean VIA heritage score $(\mathrm{M}=7.23)$. For example, the top right figure represents the relation between location and affiliation for episodes involving no food-related activity, where a participant reporting average VIA scores is using the mainstream language with an interlocutor belonging to the mainstream cultural group.

The influence of person-level variables on the relation between momentary affiliation and episode-level 
variables is best shown through the visualization of the interaction between these two levels. Figure 3 provides an example of such an interaction: the relation between language spoken and momentary affiliation during an episode, at different levels of VIA-M. This graph shows that language/affiliation patterns depend on the level of VIA-M. For people with low acculturation to the mainstream group (as denoted by a low VIA-M score), the language of interaction has relatively little importance - such people are very likely to identify with a heritage/hybrid group in all circumstances. On the other end of the continuum, for people with high acculturation to the mainstream group (as denoted by a high VIA-M score), different languages of interaction predict very different probabilities of affiliation. Heritage language is associated with a high probability of affiliating to a heritage/hybrid group, whereas mainstream language is associated with the converse. In other words, Figure 3 shows that the differential predictive power of language is greatest when overall acculturation to the mainstream group is high. Of course, other interactions between variables could have been chosen; Figure 3 only serves as an illustrative example of the influence of macro-variables on the relation between momentary affiliation and micro-variables. This finding strongly suggests that both levels are important in predicting cultural affiliation during an episode.

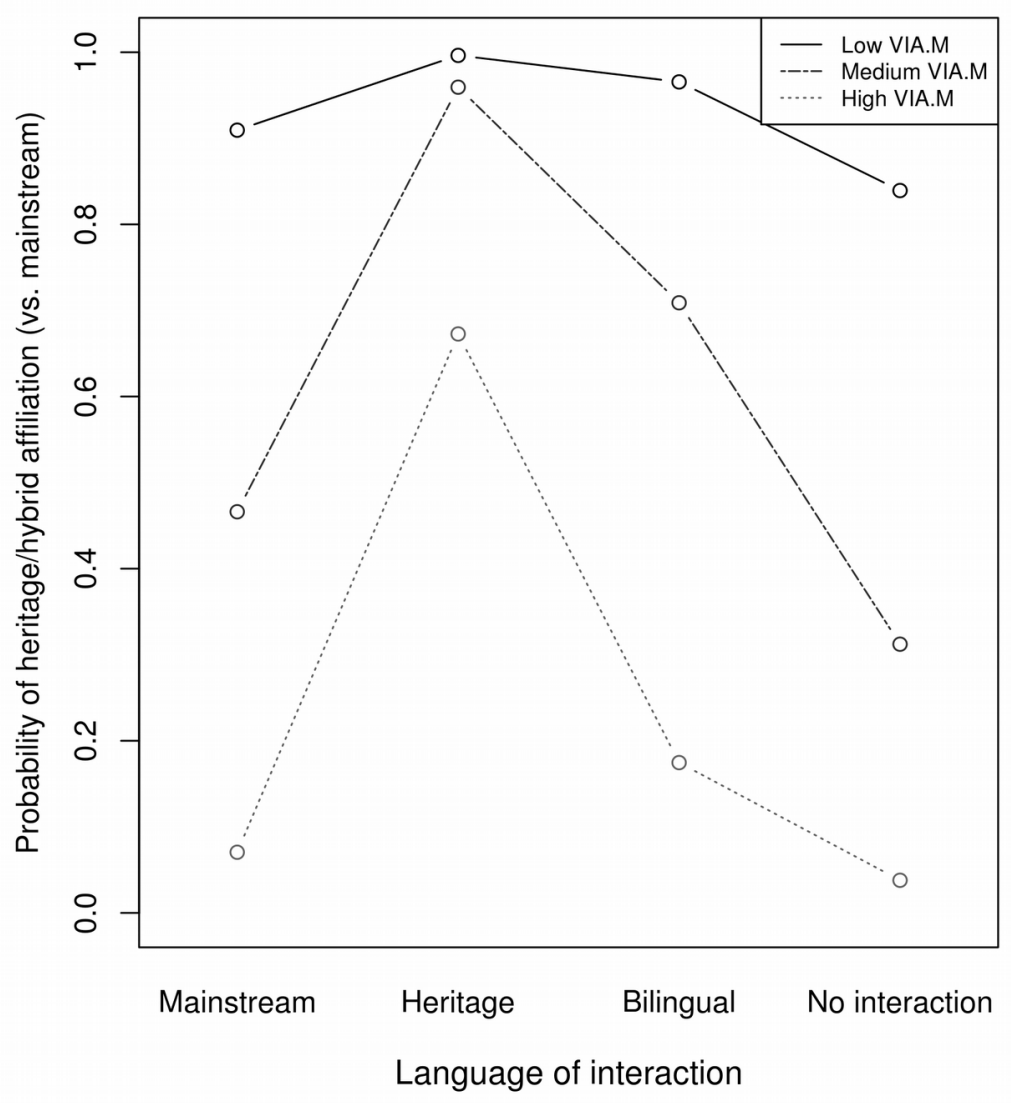

Figure 3 | Interaction between language of interaction and VIA mainstream scores in predicting heritage/hybrid vs. mainstream cultural affiliation.

The reference levels are: No food-related activity, home location, mainstream culture of interlocutor, and mean VIA heritage score $(M=7.23)$.

Predicting heritage vs. hybrid affiliation. Incremental changes in model fit as a result of hierarchical entry of predictors in our second model are presented in Table 3. Only the first two steps produced a decrease in 
information criteria values and a statistically significant chi-square value for likelihood ratio tests, indicating that the introduction of macro-variables did not increase model fit. Moreover, their introduction did not account for any intercept variance among individual people. However, the very high Somers' D final value showed that the model as a whole was effective in accurately predicting cultural affiliation. These results converge to show that general acculturation preferences do not explain variation in affiliation to heritage versus hybrid groups, but that variables characterizing the local context of an episode successfully do so by making accurate differential predictions.

Table 3 | Model Fit in Predicting Hybrid vs. Heritage Affiliation

\begin{tabular}{|c|c|c|c|c|c|c|c|}
\hline Model & $\tau^{\wedge} 00$ & Somers' D & AIC & BIC & loglik & X2diff (df) & $\mathbf{p}$ \\
\hline Null model & & & 370.4 & 378.1 & -183.2 & & \\
\hline 1. Structural context & & .80 & 359.9 & 383.2 & -174.0 & & \\
\hline $\begin{array}{l}\text { Difference between model } 1 \text { and } \\
\text { null model }\end{array}$ & & & & & & $18.49(4)$ & .001 \\
\hline 2. Social context & 16.90 & .88 & 310.8 & 357.3 & -143.4 & & \\
\hline $\begin{array}{l}\text { Difference between model } 2 \text { and } \\
\text { model } 1\end{array}$ & & & & & & $61.15(6)$ & $<.001$ \\
\hline 3. Acculturation scores & 16.77 & .88 & 314.6 & 368.9 & -143.3 & & \\
\hline $\begin{array}{l}\text { Difference between model } 3 \text { and } \\
\text { model } 2\end{array}$ & & & & & & 0.19 & .91 \\
\hline
\end{tabular}

Note. $\mathrm{AIC}=$ Akaike Information Criterion; $\mathrm{BIC}=$ Bayesian Information Criterion; loglik = log likelihood. The introduction of macro-variables reduced intercept variance by $0.01 \%$.

Table 4 reports the regression results for the full model. In accord with measures of model fit, both VIA variables have an adjusted odds ratio (OR) close to 1 and fail to reach statistical significance. Food-related activity and location are also not statistically significant in the full model, although they were at step 2 . This change from statistical significance to non-significance upon introduction of language and culture of interlocutor suggest that the variance accounted for by this characterization of structural aspects of the episode context is subsumed by social aspects of the episode. Indeed, both language and culture of interlocutor yielded statistically significant log-odds. In other words, these two variables allow for differential predictions regarding identification with heritage versus hybrid groups. Figure 4 shows the relation between levels of these two variables and cultural affiliation, with characteristics similar to those of Figure 2. In terms of language of interaction, speaking a heritage language during an episode was associated with a higher probability of affiliating to a heritage group, while there was little variation for other language levels. The cultural group label of interlocutor, as attributed by the participant, displayed a very different pattern - a hybrid cultural group label for the interlocutor predicts a markedly higher probability of activating a salient hybrid identity in the participant. These results indicate that hybrid versus heritage momentary identity can be differentially predicted from variables characterizing the local social context of an episode.

\section{General Discussion}

Conducting acculturation research in the context of Canada's multiculturalism has forced us to reconsider central tenets of the traditional acculturation research paradigm, and has thereby compelled us to consider 
innovative ways to study this multifaceted phenomenon. The C-DRM is a concrete result of this process. It aims to capture some of the complexity in the lives of multicultural people, inhabiting two or more cultural worlds, by taking local context into account and by focusing on hybrid cultural identities. The studies reported here show that the C-DRM successfully fulfills these requirements, yielding novel and nuanced results.

Table 4 | Fixed Effects and Random Effects for the Full Model Predicting Hybrid vs. Heritage Affiliation

\begin{tabular}{lllllll}
\hline \multicolumn{1}{c}{ Parameter } & Adjusted OR & Estimate & SE & $\mathbf{9 5 \%} \mathbf{C l}$ & $\mathbf{z}$ & $\mathbf{p}$ \\
\hline $\begin{array}{l}\text { Random effects } \\
\text { Intercept Variance }\end{array}$ & & & & & & \\
\hline $\begin{array}{l}\text { Fixed effects } \\
\quad \text { Intercept }\end{array}$ & 16.77 & & & & \\
$\quad$ Level 1 (episodes) & 1.03 & .03 & 4.15 & {$[-6.14,9.19]$} & 0.01 & .99 \\
Food-related activity yes & 0.62 & -0.48 & 0.55 & {$[-1.79,0.49]$} & -0.87 & .38 \\
Location school & 0.31 & -1.17 & 0.84 & {$[-3.13,0.36]$} & -1.40 & .16 \\
Location work & 0.10 & -2.28 & 1.47 & {$[-10.02,15.79]$} & -1.55 & .12 \\
Location other & 0.36 & -1.02 & 0.73 & {$[-1.89,0.95]$} & -1.40 & .16 \\
Language heritage & 106.08 & 4.66 & 1.00 & {$[2.68,9.52]$} & 4.66 & $<.001$ \\
Language bilingual & 2.55 & 0.94 & 0.85 & {$[-0.68,3.35]$} & 1.11 & .27 \\
Language no interaction & 1.83 & 0.60 & 1.29 & {$[-2.27,2.49]$} & 0.47 & .64 \\
Interlocutor heritage & 1.70 & 0.53 & 0.93 & {$[-1.21,3.41]$} & 0.57 & .57 \\
Interlocutor hybrid & 0.18 & -1.71 & 0.99 & {$[-5.01,0.57]$} & -1.73 & .08 \\
Interlocutor none & 20.76 & 3.03 & 1.29 & {$[1.63,12.58]$} & 2.34 & .02 \\
$\quad$ Level 2 (participants) & & & & & & \\
VIA-M & 1.19 & 0.17 & 0.55 & {$[-1.00,1.11]$} & 0.31 & .76 \\
VIA-H & 1.08 & 0.07 & 0.46 & {$[-0.80,0.88]$} & 0.16 & .87 \\
\hline
\end{tabular}

Note. $\mathrm{OR}=$ odds ratio; $\mathrm{Cl}=$ confidence interval; interlocutor = identified cultural group of other person(s) in the interaction.

The finding that characteristics of the local context of an episode, combined with an assessment of general acculturation attitudes, allowed us to accurately predict momentary cultural affiliation is particularly noteworthy. It underscores the fluid nature of acculturation and suggests that a systematic portrayal of the local context can at least partly account for variability in affiliation. In other words, it seems plausible that momentary cultural affiliation is the product of a complex interplay between and micro and macro factors. A corollary to this view is that subtle changes in the local environment, be they changes of location or changes in the language spoken, are associated with changes in the subjective experience of cultural identification. These results echo Hong and colleagues' (Hong et al., 2000) work on cultural frame switching, which shows that priming biculturals through the use of iconic cultural images induces changes in "culturally based interpretative lenses", as Benet-Martinez and colleagues describe it (2002, p. 492). Although the methods used in the current study did not permit us to assess momentary intrapersonal cultural changes beyond self-identification, it seems plausible that changes in cultural affiliation would be paired with other culturally relevant characteristics, such as cultural values or culturally based interpretative lenses. In this sense, the results reported here might be evidence of naturalistic 
frame switching, possibly induced by contextual cues such as location, language, or activity.
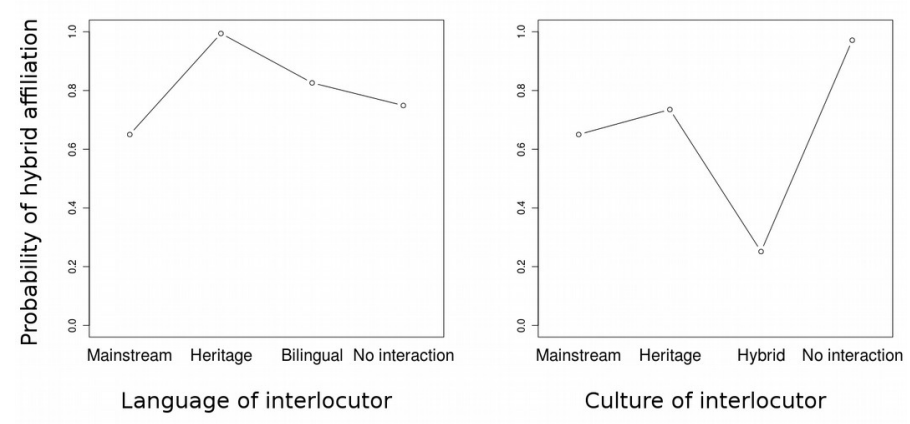

\section{Figure 4 | Probability of affiliating to hybrid vs. heritage cultural groups.}

The reference levels are: No food-related activity, home location, mainstream language of interaction, mainstream culture of interlocutor, mean VIA mainstream score $(\mathrm{M}=6.69)$, and mean VIA heritage score $(\mathrm{M}=7.23)$.

These results also suggest, however, that although cultural identification is fluid, general acculturation attitudes may impose boundaries on the extent of this malleability. Based on the pattern displayed in Figure 3, we propose that general attitudes may delineate the space in which micro-variation plays out. Conversely, it seems plausible that repeated micro-variation in one direction would push back these boundaries and thus longitudinally expand the space of possible micro-variation in cultural affiliation. In other words, repeated identification with a specific cultural group over time might eventually solidify into stable positive attitudes toward this group. This dynamic developmental interplay between micro-variation and long-term shifts in stable macro-level attitudes is only theoretical speculation at this point, but we believe it could constitute a key starting point for future empirical investigation. With that goal in mind, we might be able to draw inspiration from the developmental literature on micro-development, which specifically examines such interactions (see e.g., Granott \& Parziale, 2002).

This proposal on the interplay between micro-variation and macro-stability can potentially broaden our understanding of the concept of 'integration' by enriching Boski's (2008) five meanings of integration in acculturation psychology. The view suggested by the present results is one where integration represents an expanded field of cultural space, within which multicultural individuals fluidly shift cultural identification - and possibly cultural frames - by drawing on cultural affordances to meet the demands of specific local contexts. This view is different from attitudinal preferences for biculturalism (Boski's first meaning) and from bicultural frame switching (Boski's fourth meaning); essentially, it emerges from the dynamic interplay between these two stances.

A second set of interesting findings yielded by the C-DRM concerns the importance of hybrid identification. The results clearly show that the hybridization of culture is a real phenomenon in multiculturals' acculturation experiences, at least in our samples. Multicultural respondents report experiencing one or more hybrid cultural identifications during a typical day. Moreover, hybrid versus heritage identification can be differentially predicted by characterizing social dimensions of the local context. In our sample, hybrid identification was particularly likely when the interlocutor was perceived as also culturally hybrid. This finding suggests that hybrid cultural identification is distinct from heritage or mainstream identifications and that it might represent a qualitatively different phenomenon, rather than a mere mid-point between heritage and mainstream poles.

The scant examination of cultural hybridity in the acculturation literature precludes interpretation of our results in light of existing theory; indeed, the results reported here raise even more questions. For example, what are the attributes that lead one to perceive an interlocutor as culturally hybrid? The studies presented here cannot 
answer this question, but they do emphasize that hybridity matters in the life of multicultural people and they underscore the need for further theoretical and empirical examination of cultural hybridity.

So far, we have discussed the results of these studies and their potential importance for acculturation research. In this section, we want to reflect more generally on acculturation methods, and on the use of acculturation scales specifically. We mentioned in the introduction that mainstream acculturation research predominantly uses self-report questionnaires as research tools. Our critiques of the dominant paradigm constituted an important motivation to explore alternatives such as the C-DRM. At the same time, it is worth reiterating that our position does not represent a radical rejection of traditional acculturation instruments. A concise summary of our position is that acculturation questionnaires are necessary but not sufficient to appropriately study acculturation.

Our results corroborate this contention in several ways. First, the culture sheet showed that the average participant across both studies reported more than four self-relevant cultural groups, a number that goes beyond the capacities of any published acculturation questionnaire of which we are aware. Second, standard questionnaires on their own cannot capture the shifts in cultural affiliation that are captured by the C-DRM, nor the role of contextual factors in these shifts. Third, questionnaire scores from the VIA failed to predict hybrid versus heritage affiliation ${ }^{3}$. On the other hand, our results showed that general acculturation scores influenced the relation between local context and cultural affiliation; without information from the VIA, the picture would have been incomplete. In other words, acculturation scales have an important role to play but are inadequate on their own. We instead advocate a multi-method approach that triangulates information about stable attitudes and preferences with more malleable, context-specific, dynamic aspects of acculturation.

The C-DRM is an example of such an approach. A particular strength of this method is its potential for customization. It can easily be adapted to fit the purposes of different researchers and research questions. For instance, we mentioned the importance of domain specificity in acculturation and alluded to the work of other researchers (e.g., Arends-Toth \& van de Vijver, 2004) who share this concern. The C-DRM is an ideal tool to contribute to this body of work: episodes can easily be examined in terms of life domains with varying degrees of generality, from broader public-private distinctions to finer-grained differentiations.

The current studies should therefore be seen primarily as establishing feasibility, rather than as providing a definitive version of the C-DRM. As such, the results reported here should be interpreted with several limitations in mind. It would be advisable for future studies to examine more culturally homogeneous samples and to administer the C-DRM over several days, for two reasons. First, completing the C-DRM is a demanding task for participants. Collecting data over several days and then discarding data from the first day would circumvent the impact of a potential learning curve in completing this tool. Second, a single day may be a poor representation of the cultural life of multicultural people. In the current studies, not all cultural groups listed in participants' culture sheets were subsequently referenced during the episodes. A possible reason for this is that some of the cultural affiliations may only be enacted in special circumstances or more rarely. As such, collecting C-DRM data over several days would allow researchers to draw a finer-grained picture of the cultural aspects of participants' lived experience.

Beyond these DRM-specific considerations, it is worth reiterating that the C-DRM is only one example of a more general approach that seeks to address the challenges that acculturation research faces in hyper-diverse contexts. While we believe that this is a promising example, it is important to engage in a more fundamental discussion about what elements might characterize methods that do justice to the complexity and richness of acculturation. In other words, what ingredients would allow us to develop methods that have the potential to generate new research questions and to spur theoretical developments? In dissecting the C-DRM with these more

3 One could question the choice of the actual acculturation scale we used, but the Vancouver Index of Acculturation is a widely-used instrument that has consistently demonstrated good validity and reliability (see Huynh, Howell, \& BenetMartínez, 2009 for a relevant meta-analysis). 
fundamental considerations in mind, we wish to highlight three features: (1) customization to participants' idiosyncratic, personally relevant elements, (2) a focus on behaviours, and (3) an attempt to model between- as well as within-person variability. In our opinion, these elements are worth exploring further and are likely to make important contributions to a conversation about methods in acculturation research. We believe that such a discussion could prompt the development of a range of new approaches, which, in return, might help advance the field of acculturation.

\section{References}

Arends-Tóth, J., \& Van de Vijver, F. J. R. (2004). Domains and dimensions in acculturation: Implicit theories of Turkish-Dutch. International Journal of Intercultural Relations, 28(1), 19-35. doi:10.1016/j.ijintrel.2003.09.001

Arends-Tóth, J., \& Van de Vijver, F. J. R. (2006). Assessment of psychological acculturation. In D. L. Sam \& J. W. Berry (Eds.), The Cambridge handbook of acculturation psychology. (pp. 142-160). New York, NY: Cambridge University Press.

Bates, D. (2011). Linear mixed model implementation in lme4. Retrieved from http://cran.rproject.org/web/packages/lme4/index.html

Benet-Martínez, V., Leu, J., Lee, F., \& Morris, M. W. (2002). Negotiating Biculturalism: Cultural frame switching in biculturals with oppositional versus compatible cultural identities. Journal of Cross-Cultural Psychology, 33(5), 492-516. doi:10.1177/0022022102033005005

Berry, J. W. (2005). Acculturation: Living successfully in two cultures. International Journal of Intercultural Relations, 29(6), 697-712. doi:10.1016/j.ijintrel.2005.07.013

Bhatia, S., \& Ram, A. (2009). Theorizing identity in transnational and diaspora cultures: A critical approach to acculturation. International Journal of Intercultural Relations, 33(2), 140-149. doi:10.1016/j.ijintrel.2008.12.009

Boski, P. (2008). Five meanings of integration in acculturation research. International Journal of Intercultural Relations, 32(2), 142-153. doi:10.1016/j.ijintrel.2008.01.005

Bourhis, R. Y.(2001). Acculturation, language maintenance, and language shift. In J. Klatter-Folmer \& P. van Avermaet (Eds.), Theories on maintenance and loss of minority languages: Towards a more integrated explanatory framework (pp. 6-37). Munster, Germany \& New York, NY: Waxmann.

Bourhis, R. Y., Moïse, L. C., Perreault, S., \& Senécal, S. (1997). Towards an interactive acculturation model: A social psychological approach. International Journal of Psychology, 32(6), 369-386. doi:10.1080/002075997400629

Cabassa, L. J. (2003). Measuring acculturation: Where we are and where we need to go. Hispanic Journal of Behavioral Sciences, 25(2), 127-146. doi:10.1177/0739986303025002001

de Certeau, M. (1988). The practice of everyday life. University of California Press.

Chirkov, V. (2009). Critical psychology of acculturation: What do we study and how do we study it, when we investigate acculturation? International Journal of Intercultural Relations, 33(2), 94-105. doi:10.1016/j.ijintrel.2008.12.004

Clement, R., \& Noels, K. A. (1992). Towards a situated approach to ethnolinguistic identity: The effects of status on individuals and groups. Journal of Language and Social Psychology, 11(4), 203-232. doi:10.1177/0261927X92114002

Cleveland, M., Laroche, M., Pons, F., \& Kastoun, R. (2009). Acculturation and consumption: Textures of cultural adaptation. International Journal of Intercultural Relations, 33(3), 196-212. doi:10.1016/j.ijintrel.2008.12.008

Dere, J., Ryder, A. G., \& Kirmayer, L. J. (2010). Bidimensional measurement of acculturation in a multiethnic community sample of first-generation immigrants. Canadian Journal of Behavioural Science/Revue canadienne des sciences du comportement, 42(2), 134-138. doi:10.1037/a0016145

Downie, M., Koestner, R., ElGeledi, S., \& Cree, K. (2004). The impact of cultural internalization and integration on well-being among tricultural individuals. Personality and Social Psychology Bulletin, 30(3), 305-314.

Granott, N., \& Parziale, J. (2002). Microdevelopment: Transition processes in development and learning. Cambridge, U.K.: Cambridge University Press.

Hartzel, J., Agresti, A., \& Caffo, B. (2001). Multinomial logit random effects models. Statistical Modelling, 1(2), 81-102. doi:10.1177/1471082X0100100201

Hermans, H. J. M., \& Kempen, H. J. G. (1998). Moving cultures: The perilous problems of cultural dichotomies in a globalizing society. American Psychologist, 53(10), 1111-1120. doi:10.1037/0003-066X.53.10.1111

Highmore, B. (2002). The everyday life reader. Routledge.

Hong, Y., Morris, M. W., Chiu, C., \& Benet-Martínez, V.(2000). Multicultural minds: A dynamic constructivist approach to culture and cognition. American Psychologist, 55(7), 709-720. doi:10.1037/0003-066X.55.7.709

Huynh, Q.-L., Howell, R. T., \& Benet-Martínez, V. (2009). Reliability of bidimensional acculturation scores: A meta-analysis. Journal of Cross-Cultural Psychology, 40(2), 256-274. doi:10.1177/0022022108328919

Kahneman, D., Krueger, A. B., Schkade, D. A., Schwarz, N., \& Stone, A. A. (2004). A survey method for characterizing daily life experience: The Day Reconstruction Method. Science, 306(5702), 1776-1780. doi:10.1126/science.1103572

Kirmayer, L. J. (2013). Embracing uncertainty as a path to competence: Cultural safety, empathy, and alterity in clinical training. Culture, Medicine, and Psychiatry. Advance online publication. doi:10.1007/s11013-013-9314-2

Knabe, A., Rätzel, S., Schöb, R., \& Weimann, J. (2010). Dissatisfied with life but having a good day: Time-use and well-being of the unemployed. The Economic Journal, 120(547), 867-889. doi:10.1111/j.1468-0297.2009.02347.x

Kopperud, K. H., \& Vittersø, J. (2008). Distinctions between hedonic and eudaimonic well-being: Results from a day 
reconstruction study among Norwegian jobholders. Journal of Positive Psychology, 3(3), 174-181. doi:10.1080/17439760801999420

Lechuga, J. (2008). Is acculturation a dynamic construct?: The influence of method of priming culture on acculturation. Hispanic Journal of Behavioral Sciences, 30(3), 324-339. doi:10.1177/0739986308319570

Lefebvre, H. (2002). Critique of everyday life. New York, NY: Verso.

Malchow-Møller, N., \& Svarer, M. (2003). Estimation of the multinomial logit model with random effects. Applied Economics Letters, 10, 389-392. doi:10.1080/1350485032000082018

Moran, J. (2005). Reading the Everyday (new edition). London, U.K.: Routledge.

Navas, M., García, M. C., Sánchez, J., Rojas, A. J., Pumares, P., \& Fernández, J. S. (2005). Relative Acculturation Extended Model (RAEM): New contributions with regard to the study of acculturation. International Journal of Intercultural Relations, 29(1), 21-37. doi:10.1016/j.ijintrel.2005.04.001

Ryder, A. G., Alden, L. E., \& Paulhus, D. L. (2000). Is acculturation unidimensional or bidimensional? A head-to-head comparison in the prediction of personality, self-identity, and adjustment. Journal of Personality and Social Psychology, 79(1), 49-65. doi:10.1037/0022-3514.79.1.49

Ryder, A. G., Alden, L. E., Paulhus, D. L., \& Dere, J. (2013). Does acculturation predict interpersonal adjustment? It depends on who you talk to? International Journal of Intercultural Relations. Advance online publication. doi:10.1016/j.ijintrel.2013.02.002

Ryder, A. G., Ban, L. M., \& Chentsova-Dutton, Y.E. (2011). Towards a Cultural-Clinical Psychology. Social and Personality Psychology Compass, 5(12), 960-975. doi:10.1111/j.1751-9004.2011.00404.x

Shweder, R. A. (1990). Cultural psychology: What is it? In J. W. Stigler, R. A. Shweder, \& G. E. Herdt (Eds.), Cultural psychology: Essays on comparative human development (pp. 1-43). Cambridge, U.K.: Cambridge University Press.

Singer, J. D. (1998). Using SAS PROC MIXED to Fit Multilevel Models, Hierarchical Models, and Individual Growth Models. Journal of Educational and Behavioral Statistics, 23(4), 323-355. doi:10.3102/10769986023004323

Singer, J. D., \& Willett, J. B. (2003). Applied Longitudinal Data Analysis. Oxford, U.K.: Oxford University Press.

Somers, R. H. (1962). A new asymmetric measure of association for ordinal variables. American Sociological Review, 27(6), 799811. doi: $10.2307 / 2090408$

Van de Vijver, F. J. R., Van Hemert, D. A., \& Poortinga, Y.H. (Eds.). (2008). Multilevel analysis of individuals and cultures. New York: Lawrence Erlbaum Associates.

Vertovec, S. (2007). Super-diversity and its implications. Ethnic and Racial Studies, 30(6), 1024-1054. doi:10.1080/01419870701599465

Ward, C., \& Kus, L. (2012). Back to and beyond Berry's basics: The conceptualization, operationalization and classification of acculturation. International Journal of Intercultural Relations, 36(4), 472-485. doi:10.1016/j.ijintrel.2012.02.002

Watanabe, D. H., \& Ryder, A. G. (2007). A new approach to measuring acculturation in context: A cultural adaptation of the Day Reconstruction Method. Presented at the 68th Annual Convention of the Canadian Psychological Association (CPA), Ottawa, ON. 